\title{
ERRATUM
}

doi:10.1038/nature07833

\section{Adaptive immune features of natural killer}

\section{cells}

Joseph C. Sun, Joshua N. Beilke \& Lewis L. Lanier

Nature 457, 557-561 (2009)

In the right panel of Fig. $4 \mathrm{~b}$ of this Article the $x$-axis labels for 'Naive' and 'Memory' were inadvertently reversed in the histogram. The 'Naive' label should be on the left and 'Memory' on the right. 